\title{
Reconstructing Biological Gene Regulatory Networks: Where Optimization Meets Big Data
}

\author{
Spencer Angus Thomas • Yaochu Jin
}

Received: date / Accepted: date
Abstract To be written.
Items to address:
1) long URLs in bibliography (have been removed for the time being)
2) check run/time plot papers
3) add metaheuristic plot
4) spell check
5) check journal style eg punctuaution
6) write abstract (after paper has been finalised)
7) check refs

Keywords Metaheuristics · Evolutionary Algorithms · Optimization - Gene Regulatory Network Reconstruction · Big Data · Data Science

\section{Introduction}

Biological systems can be modelled using gene regulatory networks (GRNs), where a group of genes influence each others dynamics behaviour. GRNs are the most important organisation level within a cell [22], and are the focus of much research in the growing field of systems biology [40]. The building blocks of gene networks are not well known [43, 94, 104], however the role of each gene can be better understood by investigating their interactions and topology within GRNs [37]. Systems biology can broaden our knowledge about networks that are responsible for basic biological functions and robustness, and the causes of their breakdowns leading to disease states [62]. How cellular systems are formed from the interactions between genes, proteins and small moelcules is a major challenge for biology [19].

Spencer Angus Thomas

University of Surrey

Guildford, Surrey

Tel.: 01483686056

E-mail: s.thomas@surrey.ac.uk

Yaochu Jin

University of Surrey

Guildford, Surrey

Tel.: 01483686037

E-mail: yaochu.jin@surrey.ac.uk

\subsection{Modelling}

There are several methods for modelling GRNs. Here we breifly cover Boolean and differential equation models, for details of common techniques the reader is refered to $[7,37,44,58,109]$. Logic models, the most fundamental of which are the Boolean networks [58, 82], are a popular choice as they can give information about the network topology and are relatively simple to analyse [82]. For Boolen model of a two gene system each gene can be either active (1) or inactive (0) and interactions can be modelled using IF statements. For the GRN given in Fig. 1, a simple Boolean network would model the regulations as

$$
\begin{aligned}
& g_{1}=\left\{\begin{array}{ll}
0 & \text { if } g_{2} \text { is } 1 \\
1 & \text { if } g_{2} \text { is } 0
\end{array} \quad\right. \text { Repressor } \\
& g_{2}=\left\{\begin{array}{ll}
0 & \text { if } g_{1} \text { is } 0 \\
1 & \text { if } g_{1} \text { is } 1
\end{array} \quad\right. \text { Activator }
\end{aligned}
$$

where $g_{1}$ activates itself and $g_{2}$. This leads to a flipping of each of the genes from the inactive (0) state to the active (1) state due to the repression of $g 1$ by $g 2$ and demonstrates the importance of Boolean networks in understanding steady states and robustness in GRN [58].

More detailed models include ordinary differential equation (ODE) models, which are able to model the dynamics behaviour of each gene in the network, are commonly used [18, 97, 98, 116, 131, 133]. This increase in knowledge of the system, i.e. how the expression of each gene varies over time, comes at an increased cost in model complexity and computational run time as it requires an ODE solver. In general for an $N$ gene network the dynamic behaviour can be modelled as $d x_{N} / d t=f_{N}\left(x_{1}, x_{2}, \ldots, x_{N}\right)$, where $f_{N}$ represents the regulation between network genes, and is commonly modelled as a Hill function [55, 117-119, 126]. Hill functions are non-linear equations that are derived from Michaelis-Menten enzymatic kinetics [3, App. A]. A possible ODE model of the GRN in Fig. 1 using Hill functions and summation logic to combine the auto regulation of $g_{1}$ with the repression from $g_{2}$ would take the 
form

$$
\begin{array}{ll}
\dot{g}_{1}=\frac{w_{1}}{2}\left(\frac{\beta_{1}}{1+\left(\phi_{1} / g_{2}\right)^{n}}+\frac{\beta_{1} g_{1}^{n}}{\phi_{1}^{n}+g_{1}^{n}}\right)-\gamma_{1} g_{1} & \text { Repressor } \\
\dot{g}_{2}=w_{2} \frac{\beta_{2} g_{1}^{n}}{\phi_{2}^{n}+g_{2}^{n}}-\gamma_{2} g_{2} & \text { Activator, }
\end{array}
$$

where $w_{N}$ is the interaction weight, $\beta_{N}$ is the maximum activation, $\phi_{N}$ is the threshold for the interaction, $\gamma_{N}$ is the degradation of the protein from gene $N$, and $n$ is the Hill coefficient. Non-linear models are favoured as interactions in nature, such as gene regulation, oftern have non-linear characteristics in their behaviour [76, 123].

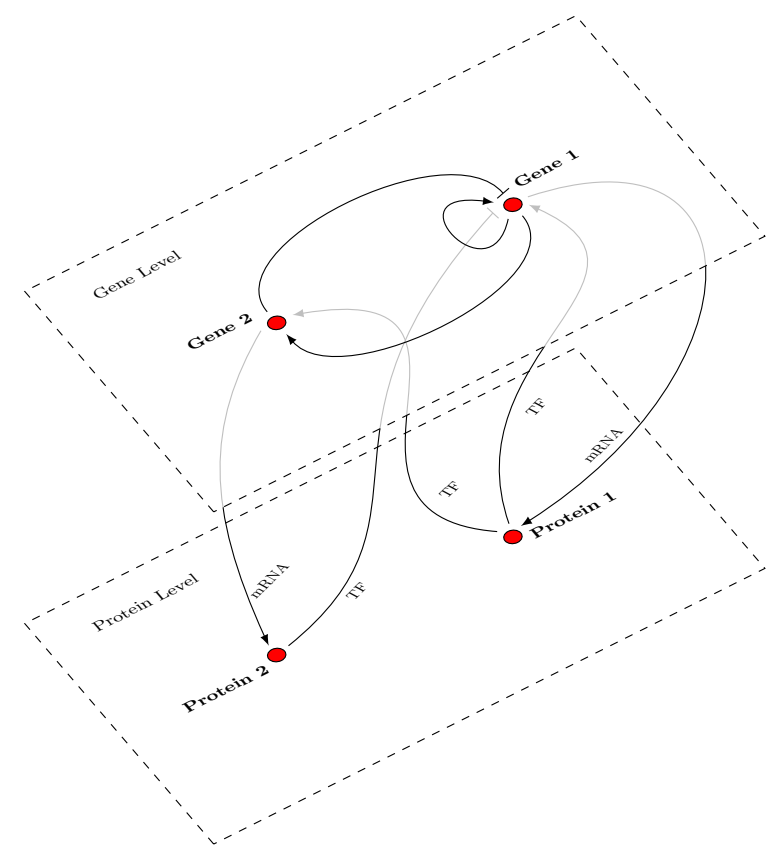

Fig. 1: a gene regulatory network model showing how regulation on the gene level corresponds to mRNA and Transcription Factor (TF) production on the Protein level

Another common ODE modelling technique is the SSystem [40, 48, 86, 87, 89, 99]. This is a power law formalism, which in general is

$\dot{x_{i}}=\alpha_{i} \prod_{j=1}^{N} x_{j}^{g_{i, j}}-\beta_{i} \prod_{j=1}^{N} x_{j}^{h_{i, j}}$.

For the GRN in Fig. 1 the S-System model would be

$$
\begin{aligned}
& \dot{x_{1}}=\alpha_{1} x_{1}^{g_{1,1}}-\beta_{1} x_{1}^{h_{1,1}} x_{2}^{h_{1,2}} \\
& \dot{x_{2}}=\alpha_{2} x_{1}^{g_{2,1}}-\beta_{2} x_{2}^{h_{2,2}}
\end{aligned}
$$

where $\alpha_{i}$ and $\beta_{i}$ are the production and degradation weights. Here we can see that $x_{1}$ self activates in the first term, while the second term combines the repression by $g_{2}$ as well as the degredation of $g_{1}$ 's mRNA. For $x_{2}$ we have a simpler equation where the first term is for the activation form $g_{1}$ and tyhe second term is for the degredation of the mRNA from $g_{2}$.

Other forms of models are based on Baye's Theorem $[20,37,100,122,132]$ which are directed graph models that display an edge to represent a causal link between two nodes. Informatics models based on Pearson's correlation [30] or mutual information [21, 80, 90, 135] use expression profiles to determine the likelyhood of a connection between two genes. Other models based on the state of a system include Petri Nets [58] and state charts [32].

\subsection{Reconstruction}

Gene regulatory networks are not fully understood [115] and the reconstruction of biological GRNs is one of the most complex tasks in bioinformatics [82]. Using expression data to reconstruct gene regulatory networks is one of the most important challenges for research in systems biology [73] and an active area of research [40]. The reverse engineering of GRNs therefore serves as an intermediate step between systems biology and bioinformatics [37]. Current high-throughput experiments can provide genome-wide gene expression measurements and an active area of research is the inference of gene networks from this data [40]. Despite experimental advances in data collection techniques, significant costs lead to limited availability for fine grain time series data for a given network. Penfold and Wild [91] noted that for microarray time series data for 3 replicates each with 25 time points costs in the region of $£ 30,000$ (over $\$ 45,000$ ). Furthermore, specific growth conditions for many organisms mean that much of the data is heterogeneous and can not necessarily be used together. This has lead to the under determinism of such problems, often referred to as 'the curse of dimensionally', where there is insufficient time series data available to statistically reconstruct large networks [40, 58, 109].

\subsection{Optimization}

As biological networks are often large [40], particularly for more complex organisms, and thus sophistocated reconstruction techniques are required. One such technique is the use of optimization algorithms to reconstruct the biological networks based on data which are often noisy and incomplete $[6,40]$, which is a general problem in biology [126]. Optimization algorithms have the advantage of not requiring prior knowledge of the system, but only an evaluation of potential solutions and are a powerful tool for modelling complex problems in biology [125]. In order to fully reconstruct a GRN, one must identify both the topology and parameterisation of the connections, resulting in a vast search space of the problems and thus making optimization algorithms an attractive method.

\subsection{Big Data}

Big data is characterised by increasing volume, variety and velocity of data generated [69], see Fig. 2, rather than simply running repeats of the same experiments producing replicate data sets. Recently data veracity (uncertianty and reliability) [111] is also used with the others to 

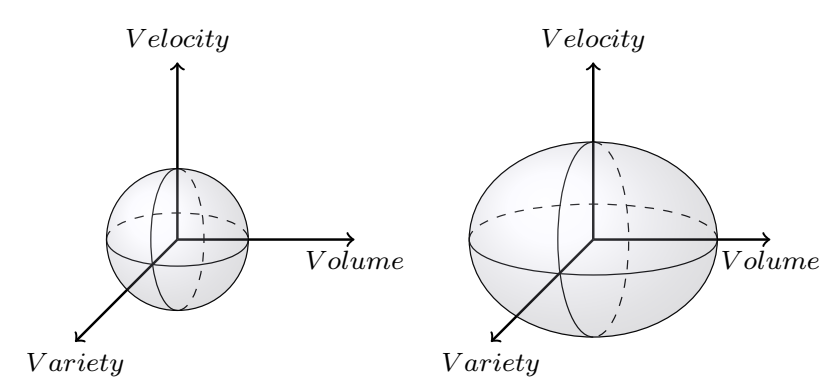

Time

Fig. 2: how data is changing: high-throughput experiments and improvements in data storage will lead to significant increases in the volume and velocity of data over the next few years. Data variety will also increase with additional types of experimental data being stored, however this will likely be at a lower rate the the others. Veracity is another aspect of big data though not shown here

form what is known as the 'Four V's of big data'. According to IBM "Every day, we create 2.5 quintillion bytes of data - so much that $90 \%$ of the data in the world today has been created in the last two years alone" [49]. This is $2.5 \times 10^{18}$ bytes per day and results in somewhere in the region of $10^{21}$ bytes of data in the world today, which is comparable with the mass of the Moon $\left(73.5 \times 10^{21} \mathrm{~kg}\right)$ [127].

With this in mind, GRNs provide a platform for a biological big data problem, and the reconstruction of these GRNs provides an overlap between biologists and computer scientists. The volume of data is ever increasing with developments in next generation sequence techniques such as high-throughput experiments. Analytical and computatutional developments will eventually allow us to collect, process and analyse data in real time increasing the data velocity. Improvements in next generation sequencing techniques will reduce noise and errors in measuremetns and help address the veracity of the data. With so many potential experimental conditions and measurement combinations, it is clear that the variety of data will grow with time. However increasing data variety means that GRN reconstruction techniques must utilise data integration methods in order to build realistic biological models. The need for data integration and 'curation' [10] will increase rapidly as the data deluge pushes biology towards the 'Fourth Pradigm' [45] as data intesive science.

\subsection{Optimization and Big Data}

Gene expression data is growing at an increasing rate and the fields of biology, systems biology and bioinformatics are entering the Fourth paradigm. As a consequces these fields will have to start dealing with the big data problems and develop universal data collection, curation, storage and retirval techniques in order to develop our understanding of GRNs during the data deluge. As mentioned, the reconstruction of GRNs from gene expression data is a platform for studying optimization techiniques and principles for real world problems. Due to the large search space, sparse and incomplete, data and potentially complex fitness landscapes, these reverse engineering problems are well suited to metaheuristic techniques. Therefore using gene expression data to reconstruct GRNs is both an optimization problem and a big data problem, and can be thought of as a Big Optimization Problem.

The paper is structured as follows; Section 2 provides a brief overview of various modelling methods for biological network reconstructions. In Section 3 we outline some metaheuristic methods for solving optimization problems such as biological networks. We discuss the role of Big Data in biological networks in Section 4. Next we given an overview of some existing reconstruction algorithms in Section 5. Finally we then provide discussion and conclusions on the topic in Section 6 .

\section{Modelling Networks}

\subsection{Models}

There are various methods one can use in order to model GRNs such as, static or dynamic, continuous or discrete, linear or non-linear, deterministic or stochastic models [37]. It is possible to model various levels of biological activity from gene regulation and protein interaction to metabolic and biochemical reaction [29]. Figure 1 shows a simplified two gene system which models the gene and protein interaction levels only. This indicates that for a large number of genes even a simplified model can quickly become complicated.

\subsection{Dealing with data}

Each data set can be either experimentally measured or synthetically generated from a target network, which is usually the goal of the reconstruction. Synthetic, or artificial data sets are used because the limited availability and cost of experimental data. However it also allows comparisons between the predicted network and the actual known network to be made and thus assess the reliability and performance of the reconstruction method.

Due to the complexity of experimental data sets, artificial data is not a realistic representation of biological data [37], thus varying levels of noise are often added to the data to make it more 'realistic' [37, 107], as well as to further test the algorithms performance [89]. When using artificial data, it is also possible to produce many time points and replicate data sets to aide the reconstruction process [7, 37, 91, 107].

Those dealing with real data sets are often required to maximise the amount of data through interpolation of the avaialble data [106]. This has the added benefit of giving constant time intervals for the data points, as experimental time series may not have constant time steps throughtout the measurements [59].

For a large networks it is possible to cluster genes with similar expression profiles together to reduce the size of 
the network and reduce the dimensionality of the problem. This method has been used by several researchers [29, 47, 120], however this adds the need for a clustering process and averaging expression profiles before the optimization stage creating additional overhead and potential errors. Altough this techniques does fit with some biological observations, i.e. sparcity, it is still a simplification.

Additional information can be taken other types of data sets, such as from knockout and pertubation experiments. The former removes a gene from a genome, known as a null or mutant strain, and compares expression levels of genes with 'wild type' organism [2]. This process is known as differential gene expression and can give significant insight to which genes are in the same network and thus affected by the deletion. The latter gives more fine detailed information into the interconnectivity in the network by varying the state of a particular gene to observe any changes in other genes in the network [7].

\subsection{Topological Networks}

Topological models are of particular important to our understanding of the behaviour of GRNs due to the modularity of biological systems and the functions of these modules such as AND and OR gates for time delays and robustness $[4,75,83,110]$. Topological models can also help identify auto regulation of genes, which are known for their functional roles such as decreasing response time or enhanced variation in expression levels [4].

Swain et al. [115] illustrated the importance of topology by using a caterpillar and butterfly analogy, where the two insects contain the same genes, the connectivity of which is changed during the crystalline phase resulting in the physical difference between the two. In some cases the topology of the network is more important than the parameterisation as the structure can determine the dynamic behaviour of the network [40, 115]. For some cases different parameters settings in network can lead to the same behaviour for small network motifs, for example some network motifs that produce oscillations and toggle switches contain many local parameter optima that produce the same network dynamics [117-119]. The importance of topology, over parameterisation, is also present in more complex models, such as the Drosophila segment polarity network. This model contains 48 free parameters, which when randomised, each had a $90 \%$ of being compatible to the desired behaviour regardless of parameter magnitude or range [23].

The main issue with topological models is the need for a quantitative metric for comparison between models [126]. One can use measures such as specificity, sensitivity, precision and recall, however for the practical case of an unknown topology, theses measures are useless. However for competing models of the same unknown system the fit to the experimental data can be used as a measure of model quality. For models of similar fits to the data, the simpler model, i.e. less nodes and/or connections, is preferred as it is easier to understand and less prone to over fitting [58], an example of Occam's Razor.

\subsection{Parameterised Networks}

The parameterisation of a network is also important as it allows us to investigate the modelling of the connections within a GRN. The difference between a simple linear connection and a more complex non-linear connection between genes could significantly affect their dynamics behaviour. This also gives a platform for comparing regulation type, ie activating or repressive, as well as regulation strength. Gonze [36] observed regions in the parameter space that determined the dynmaic behaviour of a fixed network structure and determined the bifurcation values for changing the newtork dynamics. Similarly in [118] the authors observed different dynamic behaviour for a fixed network by varying the parameters of one of the connections.

\subsection{Combining Topology and Parameterisation}

Both topology and parameterisation are improtant as discussed above and for complex systems, both are likely to affect the behavious of the network. In [119] it was observed that for a given topology the same behaviour was observed for multiple parameter values, however by reversing the direction of a single connection the network appear to be dependent on parameterisation. Here the authors observed a 'weak' bifurcation point in a single parameter that caused a change in the dynamic behaviour of the network in most cases.

\section{Metaheuristic Methods}

Metaheuristic methods can be used to solve difficult optimization problems with little or no prior knowledge. These are able to solve underdetermined problems [84, 109] such as the reverse engineering of GRNs. Although metaheuristic searches do not always yield the most optimum solution, they can provide a feasible solution given the problem constraints. As many metaheuristics are stochastic, it is possible to average results over numerous simulations or to find the optimum solution.

Reverse engineering GRNs is a difficult task hindered by the complexity of biological networks [33]. Both the reconstruction of the network topology and the interactions between network nodes are suited to optimization, and as a complex real world problem with a large search space contain many local optima [6]. Several optimization techniques have been used for reconstructing biological networks, however they are limited by the amount of data available and the high dimensionality of the problem [96], as well as computational power required for large networks.

\subsection{Nature Inspired Optimization Algorithms}

Evolutionary algorithms (EAs) are a class of metaheuristic algorithms that are based on the process of Darwinian evolution where the fittest solutions survive. These algorithms are able to deal with large search spaces [82] and 


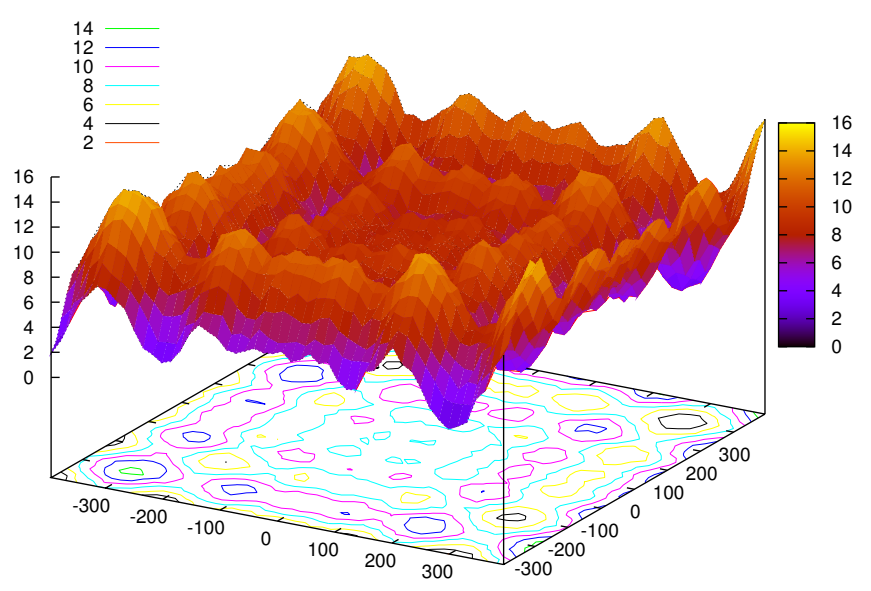

Fig. 3: complex fitness landscape based on the Schwefel function [102] containing many local minima

complex fitness landscapes, such as Fig. 3, and are therefore well suited to network reconstruction problems [115]. In these algorithms, a possible solution to the optimization problem is represented as an individual, which is in a population of individuals. Through operations based on crossover and mutations, a 'parent' population is used to produce an 'offspring' population with each individual's fitness determined by evaluating an objective function of the optimization problem. Individuals are then selected based on their fitness, with the solutions satisfying the objective function the most being the fittest solutions and are used as the parent population for the next generation. This process is repeated until some stopping criteria is met, either the desired number of generations or some threshold for the objective function.

The genetic algorithm (GA) and evolution strategy (ES) are two common types of EAs which are used in a variety of optimization problems. Historically GAs were for binary valued optimization problems and ES only used the mutation operation. Now however, there are many real valued GAs, most notably NSGA-II [26], and ESs that use crossover operations making an ES and a real valued GA very similar [25, pp.133]. Another common EA is a genetic programming (GP) where each individual is an evolvable 'tree-like' structure and solutions are oftern not of the same form, see Fig. 4.

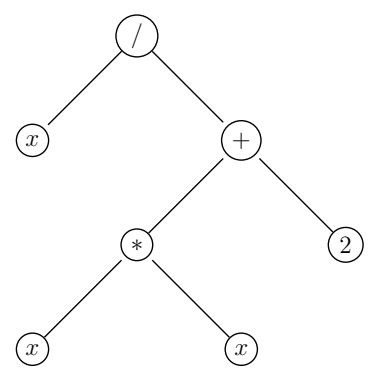

(a) $x /\left(x^{2}+2\right)$

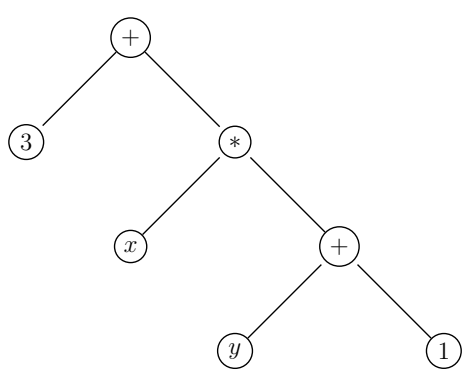

(b) $3+x(y+1)$
Fig. 4: example of individuals in a genetic program
Other forms metaheuristics that are inspired by nature are a group of algorithms known as swarm intelligence, the most common of which is the particle swarm optimization (PSO) [60]. Here each particle in a swarm represents a candidate solution for the optimization problem. As with EAs, these solutions are evaluated based on an objective function and then ranked in order of how well they meet the criteria of the objective. Each of the particles have an associated position and velocity which are updated based on the position and velocity of the local best and global solutions from the previous generation, see Fig. 5. Other notable swarm intelligence algorithms are ant colony optimization (ACO) [28] and bee colony optimization (BCO) [57].

Another optimization process that is inspired by nature is simulated annealing, where the physical process of slowly cooling a melted substance to form a crystalline structure, known as annealing, is adapted to solve optimization problems [64]. Here a slower rate of cooling causes larger decrease in the materials thermodynamic free energy. Simulated annealing mirrors this by slowly reducing the probability of poor solutions, which are needed for escape local optimum, being accepted into the population.

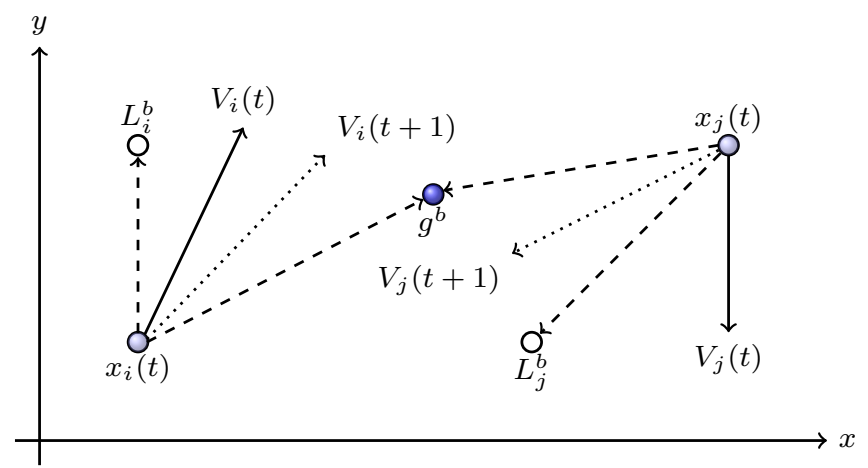

Fig. 5: an Example of two individual particles, $x_{i}$ and $x_{j}$, at time $t$ in a particle swarm optimization algorithm. Shown is how the velocity of a particle changes over time based on the position of the best local $\left(L^{b}\right)$ and best global $\left(g^{b}\right)$ solutions

\subsection{Other Forms of Optimization}

Other, non-nature inspired, forms of optimization include local search algorithms, that search a solutions neighbours by making slight variations to the decision variables in an attempt to improve the overall objective function. These types of searches however often lead to local optima rather than global optimal solutions as they are unable to vary solutions enough to escape the local optima, an example of this is the hill climber algorithm. Some more sophisticated local searches have been proposed such as a tabu search which remember solutions that have been previously searched and assigns them as forbidden, or tabu [35]. This allows searches to avoid local optima compared with other local searches. 


\subsection{Elitism}

The role of elitist selection in optimization is a debated issue due to its ability to aide algorithm convergence [54, 117-119] but also leading to local, rather than global, optimal solutions [12]. Reverse engineering biological networks in general have a large optimization search space as a result of the systems complexity, and even the smallest known genome can contain 200,000 interactions, after making many false simplifications [40]. If you modelled a cell through all its significant interacting constituents, the resulting complexity would be 'daunting' [61]. Due to such large search spaces, many researchers have used elitism in their reconstruction algorithms to aide the evolutionary search and reduce the computation time [48, 74, 82].

\subsection{The Number of Objectives}

\subsubsection{Single Objective}

Optimization algorithms are designed to solve a problem based on an objective function. If there is only one objective, such as minimise error then this is a single objective problem. A common objective for biological network reconstruction is simply to minimise the error between the model output and the data you are simulating [89, 97, 109, 122, 130] which can be applied to both real and Boolean networks [40]. This method can work well for small networks, however, this can lead to over fitting and many false positive connections if there is no constraint on number of connections between nodes. This becomes a problem of larger networks, not only for biological relevance but it also increases computation time dramatically. Other objective functions have been suggested such as information criterions [74, 89, 105] and the inclusion of penalty terms to reduce over fitting [21, 89, 97]. Some authors have integrated prior biological information to aide the reconstruction process $[34,76]$.

\subsubsection{Multi-objecitve}

The number of objectives used is also an area of interest in optimization applications [46, 92], such as minimising error and increasing sparsity, yielding a multi-objective real world problem. Furthermore, several combinations of these objectives can be used, for example error between the model and the data, sparsity, robustness, number of regulators, maximum connectivity of each node, regulation density, modularity, regulation chain length, biological plausibility [30], etc. Thus the use of mutliple objective functions can be used to infer an accurate network model based on the data, while also using another objective to maintain biological plausibillity, as they are able to deal with more complex models [46]. Multiobjective optimization also provides several potential solutions taken from the Pareto front and can be comapred and selected by the user based on some preference. Several multi-objective optimization algorithms are available, such as GAs for binary [114] and real valued problems [26], Predator-Prey ES [71], and Pareto-Achived ES [66], all of which are detailed in [25].

\subsubsection{Many-objectives}

With increasing number if objectives, current optimization algorithms are severily hinder and lead to many nonoptimal solutions [50]. If there are more than 3 objectives to optimization the problem is known as a manyobjective problem, an increasing area of research in the field of optimization [51]. It is possible to use several objectives simultaneously to reconstruct a GRN, as mentioned above, to develop a more realistic model. Several algorithms have been proposed to deal with more than 3 objectives, such as MOPSO [5], NSGA-III [52], GDE3 [68], MOEA/D [134], IBEA [138], and Borg (a framework) [39].

\subsection{Multiobjectivization}

With all the combinations of objective functions mentioned above it may be possibel to untilisze the observed phenomenone of multiobjectivization $[15,41,78]$ which can aide the optimization search by increasing convergence speed and obtaining global optimum [41, 42]. Multiobjectivization can be achieved by decomposing a single objective in to multiple objectives with similar goals $[14,67]$, or through the use of additional 'helper' objectives $[14,53]$ and may also provide more non-dominated solutions with no extra cost to functional evaluation [42]. However multiobjectivization can also hinder the evolutionary search and performance may be problem specfic $[14,42]$. Furthermore it has also be observed that equivalent objective functions in different domains lead to either evolutionary converegence speedup or hinder the search [117]. Multiobjectivization has been shown to speed up convergence times of inferecne algorithms, however the practicality of this application to larger networks still requires investigation [107].

\subsection{Innovization}

Innovization [27] is a process whereby innovative ideas can be found through optimization by analysing the Pareto optimal solutions are observing there special features and commonalities. It is possible to use this technique to discover new and interesting properties of a system through optimization. In [46] the authors state that multi-objective optimization can lead to the the discovery of patterns in an organisms structure so is an example of innovization in the reconstruction of GRNs. This parallels are area of big data where analysis of large data sets can provide novel information and may lead to the discovery of new laws or principles. The common example of this is Kepler's Law of planetary motion which were discovered through analysis of Tycho Brahe's systematic astronomical observations, i.e. data analysis [45]. Systems biologists can use multiobjective optimization and innovation to provide novel insights into the structure and cahracteristics of GRNs using large scale data analytics. Here we can see that the study of GRNs can act as a platform of both optimization and data science. 


\subsection{Hybrid Algorithms}

Due to the large search space of optimizing a models topology and parameters together, several hybrid algorithms have been suggested to separate theses optimization problems. Splitting these process greatly reduce the dimensionality of the problem [6] and can lead to improved algorithm performance and fitness values [74]. Current hybrid algorithms include an artificial neural network and a GA [59], a GP-PSO hybrid [16, 17], a memetic algorithm consiting of a GA and an ES [113], and differential evolution with a local search [89]. In these algorithms the first stage determines the topology of the network and the second determines the parameters of the system. In a similar methodology, nested optimization is also used to separate structure and parameter optimization [109].

\section{Big Data in Biology}

High-throughput experiments can collect vast amounts of information about an organism, not only gene expression but also experimental conditions, which can help improve the reconstruction of networks and enable the development of more realistic biological models [109]. Each experiment can produce gene expression levels for thousands of genes at a given time after some biological event giving a genome-wide view of gene expression for the first time [40]. Although currently microarray experiments are expensive and noisy [81], with improvements in technology and process the constraint of cost will decreases, and the number of time steps, as well as the frequency, will increase. Many such experiments use biological replicates, where identical strains of an organism are grown alongside each other under the same conditions to remove experimental noise. We will soon therefore, have many time points for several biological replicates for each of the genes in an organism for the specific growth conditions, i.e homogeneous data. However, with growth conditions determining the properties of the organism, numerous growth conditions are likely to be measured leading to many different heterogenous data sets, therefore our ability to combine data sets whilst reducing problems such as heterogenous noise has to improve [109].

Analysing such large quantities of gene expression data is not trivial due to their high dimensionally and noise levels [109], and biology rapidly becoming quantitative [7], data intensive, science. In order to reconstruct biological networks of significant size and complexity we will need to be able to store, use, share and analyse data efficiently. The so-called curation stage will become increasingly important as high-throughput experiments produce massive quantities of data with varying provenance. At present there is not enough detailed information to accompany the hugh amounts of data being collected [40], however, as more effort is made in data curation we will be better able to cope with the data deluge. If we are able to record vast quantities of data, along with relavent information i.e. provenance, in a usable and accessible way, systems biology can not only predict network structures, but also determine between competing models and de-

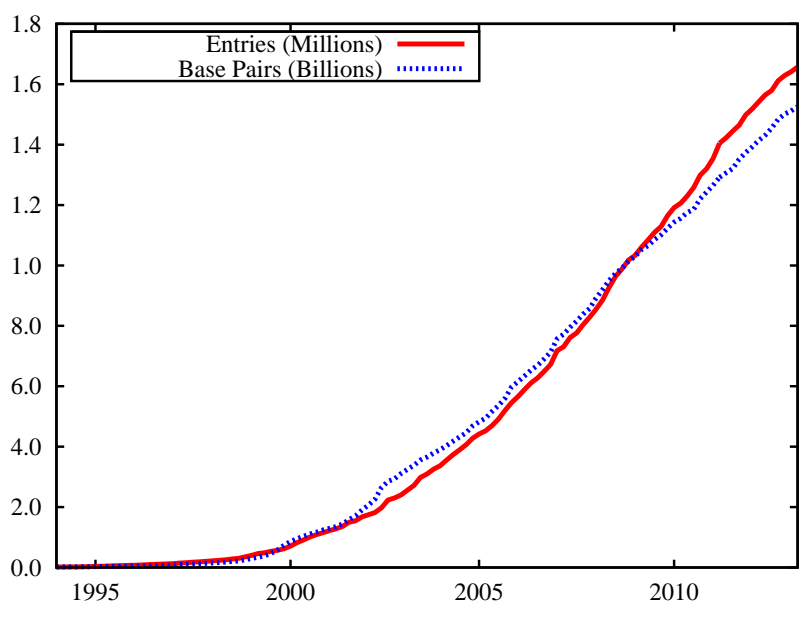

Fig. 6: number of entries to GenBank [13] database and base pairs contained, which doubles every 18 months [13]. Note the solid red line is is units of millions and the dotted blue line is in units of billions. The big challenges of big data paper has similar plot

velop our understanding of the underlying process of the complex system [128].

Many areas of small scale biology are currently going through the data deluges. The database GenBank which is doubling its number of entries roughly every 18 months [13] and the number of entries and base pairs is shown in Fig. 6. Integration techniques will allow us to combine vast amounts of information from different areas of biology and micro-biology and may lead to a unified model of biological systems.

\subsection{Big Data and Gene Expression}

Gene expression data is a practical example of big data in a real world setting. It is obvious how next generation sequencing techniques such as high-throughput experiments lead to increases in the volume of data, beyond more time course data, there is the possibility of replicates, differences in experimental conditions, as well as other organisms in the Genus when comparing similarities across Species. What may be less clear is how this data fits the other of the so called 'Four V's of Big Data'.

\subsubsection{Veracity}

How accurate, precise or reliable the data is, data veracity, will depend on the techniques used to conduct the experiments and will undoubtably improve with the techniques themselves. Biological replicates are used to reduce experimental noise [133] and remove fluctuations from the noisy techniques such as DNA arrays [59]. This in effect is improving the veracity of the data and it is possible to combine data from different high-throughput sources to increase confidence in the data used. 


\subsubsection{Variety}

Aside from obviously different types of data such as steadystate and dynamics data, gene expression experiments rarely have the same growth conditions and thus produce heterogeneous data sets. However this does provide additional data, increasing data volume, which can be used to test and compare models and may help determine biologically plausible models from those that overfit the data. Data variety is also significantly increased through the use of deletion data, where a gene is deleted in order to determine its direct regulatory targets [2, 131] and are further examples of heterogeneous data sets that can be integrated in order to learn more about an organisms GRN structure [131]. Perturbation data is also useful in reconstruction of GRNs [33] as it gives detailed information on more complex or weaker interactions [131].

\subsubsection{Velocity}

This aspect of gene expression data comes from two main areas, measurement and analysis. The measurement side includes the experimentation, where high-throughout experiments are occurring in many laboratories leading to a continual increase in the data becoming available. However, the main aspect of the data velocity comes from the analysis of this data, which is still a developing research area. The analysis ranges from turning the raw data into gene expression profiles to using these profiles to infer network structure. The latter is still in its infancy, but in the future we may be able to combine reconstruction algorithms with the experimentation to determine the structure of an organise in real time alongside the initial data analysis. Further still we can look at how models built from the data being collected compares to a those built from availabel data to test the impact of the data begin collected on model inference.

\subsubsection{Volume}

In this case the volume of data is linked to the variety and veracity of the data as demonstrated in Fig. 7. Data available on gene expression is increasing at an exponential rate [129]. Beyond biological replicates, repeat experiments, independent verification and simply more time points in the measurements add to increasing volumes of data.

\subsubsection{Linking the Four V's}

There is much overlap between the Four V's of Big Data indicated in the above paragraphs, and changes in one of them can lead to changes in at least one of the others. This implies that Big Data is more than just a large amount of data points and that several, if not all, of the asspect of Big Data are linked. It is possible to think of Big Data as a 3D surface of volume, velocity and variety, as in Fig. 2 each point with an element of veracity.

\subsection{Data Integration}

Data integration itself is an important issue in biology and is generating considerable interest [40, 45, 69], particularly heterogeneous data $[106,109]$. Heterogenous data is ubiquitous in nature due to the complexity of biology and the lack of standardised experimental protocol, resulting in incompatible sources from different data formats [136, pp. 49]. The benefit of models based on multiple data sources was demonstrated in [108], where models were less prone to over-fitting and more robust to noise and parameter perturbation, and although this increases the computational complexity, it does help reduce the problem of under-determinism [109].

Data integration is vital in data science and the reconstruction of GRNs is already beginning to combine different types of data in order to build more reliable models, such as deleted and perturbed data sets [131], and dynamic and steady-state data [137]. Information on biological sparsity [96], the number of network regulators [72], and a shallow architecture with regulators typically one step away from their targets to increase response time [4], can be used as additional objectives or as system constriants. The latter can help reduce the search space to only biologically plausible regions during the optimization and the former can potentially be used to increase the algorithms convergence via multiobjectivization as mentioned in Section 3.5.

Data provence is also an important aspect of big data and is necessary to reverse engineer biologically plausible networks, particularly when integrating data sets and assesing data veracity. More generally, for different species containing similar network structures and regulators [77], one can integrate data on similar organisms to generalise certain fundemental biological process.

These elements of gene expression data highlight the link from GRNs to big data and the need to incorporate large scale data analytics in order to deal with the 'flood' of data [123]. The curation and integration of data is a growing challenge with the rapid development of many sequencing technologies [136, pp. 51]. The comments on variety alone clearly illustrate the need for improvements in data integration, curation and analysis. Biology is in the midst of a data deluge, gene expression data is a clear example of this and Fig. 7 shows how the different types of experiments from gene expression measurements are leading to changes in data volume, variety and veracity.

\subsection{Big Data and Metaheuristics}

Metaheuristics are suited to dealling with Big Data, as they can deal will multiple objectives and constriants based on data provenance or heterogenous sources, and they are also able to deal with large search spaces which are likely to be the case with Big Data problems, such as inference of GRNs. Metaheuristcs are felxible with many kinds of algorithms and frameworks providing scope for tailoring algorithms that are better suited to certian kinds of problems. Also developments in the area of so called 'hyper-heuristics' provide a platform for evealuating dif- 


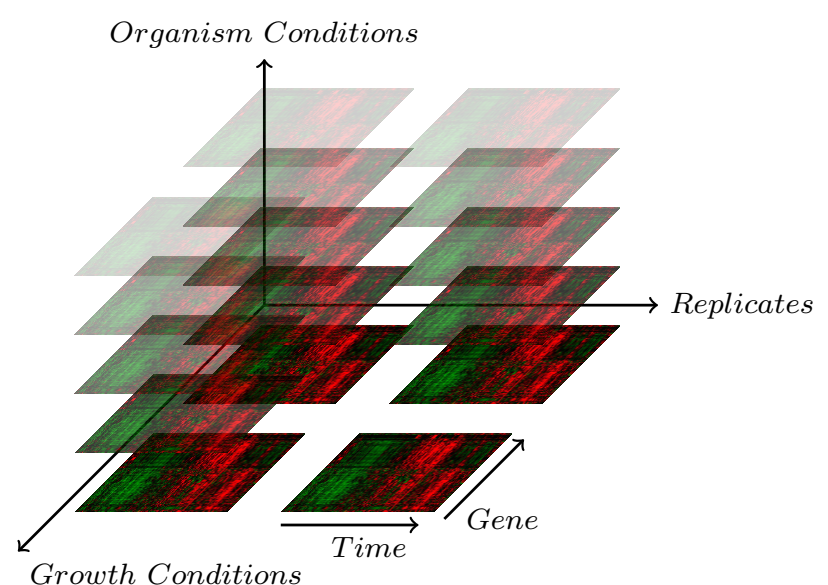

Fig. 7: how mircoarray data is big data. MReplicates provide homogenous data sets which can help reduce the veracity of the data. Heterogenous data sets are provided by varying the growth conditions of organism, i.e. the nutrients, and also by varying the organism conditions via perturbation and knockout experiments. The heterogenous data sets are examples of data variety (see text for details). An increase along any axis leads to an increase in data volume and as techniques improve indicating a link between the elements of the Four V's

ferent metaheuristics for a certian problem. More specifically, Big Data problems require researchers to improve data analytics and infer laws or principles from data, similar to Kepler and the laws of planetary motion. A type of metaheuristic, genetic programs, already been proven to infer some of the laws of physics from experimental data [101] and so provide direct evidence that metaheuristics can be used as analystical tools in big data problems. Further evidence is provided in Section 3.6, where multi-objective optimization can lead to innovative ideas through innovization. These innovations can discover special features and commonalities between Pareto optimal solutions which may be of great interest to engineering and design disciplins [27].

\section{Current Reconstruction Algorithms}

There are many reconstruction algorithms available and several reviews comparing them with different data sets, some of which are detailed in Table 1. In [7] several algorithms were tested against steady-state and dynamic data sets of varying sizes, however none of the algorithms tested were able to reconstruct networks from dynamic time-series data, of any size, significantly better than random. The reconstruction algorithms in [37] used various sized networks and concluded that none of the algorithms tested out perform the others and that not only were none of them were able to reconstruct the true network for all data sets, but all methods had a low precision and result in many false positives. In [107] it was noted EA methods are favoured due to the lack of data, however hybrid algorithms are needed for larger networks. The authors also stated that as hybrid methods are computational expensive, parallel implementation is vital. Pen- fold and Wild [91] found that reconstruction from time course was nearly always better than those from systematic knockout experiments. They also noted that NDS methods performed the best based on time series data, however they scale with the number of obsevations and are impractical for cases where many time courses are generated. This becomes particuclarly problematic when the networks are large, for 100 genes with 21 time points and 10 replicates (totalling 210 observations per gene), NDS methods require $48 \mathrm{hrs}$ of computational time per gene [91].

The is an urgent need for reconstruction tools [74], particularly those that can deal with a large number of genes and can reverse engineer networks based on real data. Many algorithms use artificial data as a benchmarking tool as the true network is known and the performance can be quantified. Although the artificial data can be useful for comparing algorithms and see how models use the data [93], they are not as complex as real biological data [37]. These benchmark tests often use multiple data sets that increase with the number of genes in the target network, however the expense of current experiments $[6,91]$ is not realistic.

Performance of reconstruction algorithms will improve with addtional data, however, advances in reverse engineering algorithms is desperately needed to incorperate data integration techniques. This may help reduce the problem of underdeterminism and allow the algorithms to cope with the high levels of noise within mircorarray data sets, as well as any missing data points.

Figure 8 shows how the computational time for network reconstruction increases with increasing network size for several data and model types. Particularly notable is the large runtimes for real data sets and the significant spread of the runtimes for networks of the same size. This spread is likely to be due to the quality of the data available, which will vary significantly between experiments and will not be present in artificially generated data sets.

Table 1: Current reconstruction algorithms. Model type acronyms are ordinary differential equation (ODE), dynamic Bayesian network (DBN) and non-linear dynamical systems (NDS).

\begin{tabular}{llll}
\hline Algorithm & Model type & Ref. & Reviewed \\
\hline ARACNE & Relevance network & {$[80]$} & {$[7,37]$} \\
Banjo & Bayesian network & {$[132]$} & {$[7,37]$} \\
NIR/MNI & ODE & {$[11,33]$} & {$[7]$} \\
GNRevealer & Neural network & {$[38]$} & {$[37]$} \\
LDST & State space & {$[95]$} & {$[37]$} \\
GeneNet & Graphical Gaussain & {$[100]$} & {$[37]$} \\
ParCorA & Pearson / Spearman & {$[24]$} & {$[37]$} \\
DE+AIC & ODE & {$[88,89]$} & {$[107]$} \\
GA+ANN & ODE + neural network & {$[59]$} & {$[107]$} \\
GLSDC & ODE & {$[121]$} & {$[107]$} \\
PEACE1 & ODE & {$[63]$} & {$[107]$} \\
GA+ES & ODE & {$[112]$} & {$[107]$} \\
G1DBn & DBN & {$[31]$} & {$[91]$} \\
VBSSM & DBN & {$[9]$} & {$[91]$} \\
TSNI & ODE & {$[1]$} & {$[91]$} \\
GP4GRN & NDS & {$[65,91]$} & {$[91]$} \\
CSI & NDS & {$[103]$} & {$[91]$} \\
GCCA & Granger causality & & \\
\hline
\end{tabular}




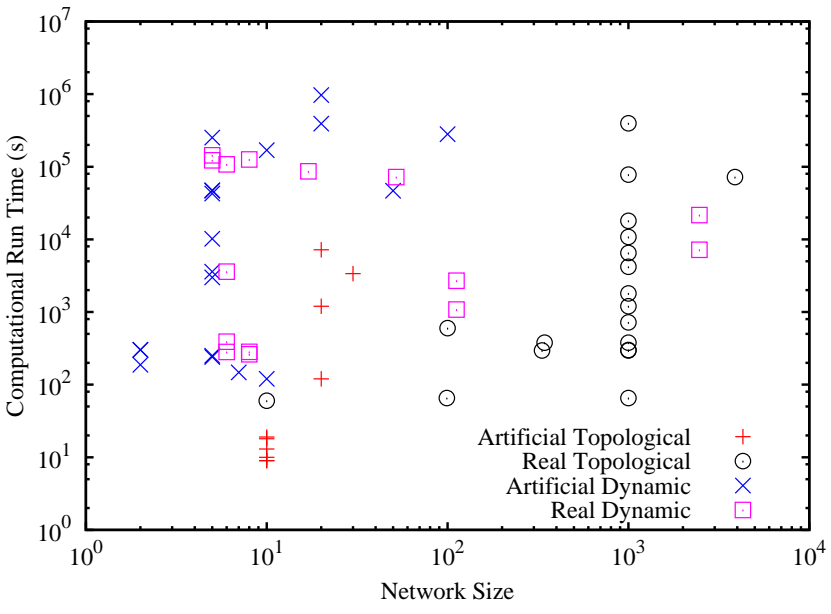

Fig. 8: reconstructed network size versus computational runtime. Data types are either from synthetic networks (Artificial) or from biological experiments (Real) and are either dynamics or topological models as indicated in the figure key. Data collected from [7, 56, 59, 79, 85, 87-89, $100,107,122,129-132]$

Model complexity is also a factor as this can significnalty effect the compuatational run time. It is also clear that reconstruction based on dynamic data sets, even for small networks are very computationally expensive. Many authors do not include information about the runtimes of their algorithms so the available information is relatively small. It does however illustrate the need to improvements in computational techniques, not only in the reconstruction algorithms and methods, but in the execution of the algorithm and other computational tools [58]. There is little research into reconstruction of GRNs based on real data compared to artificial data [40], which are ultimately just benckmarks for algorithms but are not adding to out understanding of biological networks.

\section{Discussions and Conclusions}

Parallel computing is an obvious solution for the runtimes of algorithms which can be significantly reduced by splitting them over several cores. This does, however, require some expertise and programming at a low level of abstraction [70] making the algorithm more problem specific and less useful for general reverse engineering of networks. Xiao et al. in [129] used parallel processing to reconstruct a network of 1000 genes based on artificial perturbation data and observed a parallel speedup of 20 times with 32 cores. Although they noticed a sharp decrease in parallel efficiency when using 4 cores, due to information sharing, there is little change between 4 and 32 cores. Even with this continual decrease in parallel efficiency with additional nodes, the authors reduced the computational run time from 108 mins to 5 mins.

This speedup can be furthered using graphic processing units (GPUs) can contain 1000s of cores and reduce a runtime of days into minutes, though this requires even more expertise than standard parallel computing. Despite the barrier of knowledge, parallel computing is becoming a necessity [107] for network reconstruction and as the quantity, and quality, of data increases, so must our computational abilities. Some reconstruction methods already take hours to reconstruct small networks [63, 87, 89] and others are already running on computer clusters [131]. Methods that are not intrinsically parallel, such as the S-System Eq. (3), can be decoupled so that each connection can be treated seperatly allowing parallelisation. Such techniques can significantly reduces the search space [59, 89, 109, 124].

Further computational improvements can be made at the algorithm level. As available data increases and data integration techniques improve and become more common, statistical based reconstruction methods will benefit from increasing levels of usable data. However how the algorithms deal with data and the rate at which information is processed and then used in the reconstruction needs to improve so that large and more complex networks can be reconstructed. Improvements in efficientcy in algorithm operations, such as solution evaluation and selection may lead to significant speedups.

Although the problem of under-determinism of GRNs is addressed with increasing the amount of data, metaheuristics are still an attractive option due to their flexibility and search power. Even in an ideal case were high quality time series data is plentiful, there may still be a considerable search space for connection parameters, and even for the form of connection between genes. Not only can metaheuristics be used in connection topology and parameterisation of a network, they also offer a platform for optimizing biological objectives whilst also being intrinsically parallel [109]. Several objective functions can be compared and used together to investigate their effects on the final network which may help to identify important biological objectives in addition to the potential benefits of multiobjectivization. Furthermore, with the development of many-objective optimization, there is potential for using several objectives to help steer the search towards biologically plausible solutions.

In [124] the authors noted that $95 \%$ of the optimization time for larger networks was spent on numerical integration. This can oftern lead to non-convergence of even algorithm failure [123]. However does indicating the potential for significant improvements to runtime efficiency providing improvements to the mathematical and computational techniques occur, i.e. parallelising integration calculations for newtork reconstruction.

Reconstruction algorithms must be able to scale up to 10s of thousands of genes [120] in order to model higher level organisms. The future of reconstruction algorithms is likely to contain a synergy of data intensive and optimization processes in order to analyse the data required for the optimization of a large networks search space. This Big Data optimization synergy requires both biologists and computer scientists to work together and share not only data, but also expertise, knowledge and processes.

Acknowledgements This work was funded by an Engineering and Physics Science Research Council (EPSRC) Doctoral Trainning Centre (DTC) studentship at the University of Surrey. 


\section{References}

1. Äijö, T., Lähdesmäki, H.: Learning gene regulatory networks from gene expression measurements using non-parametric molecular kinetics. Bioinformatics 25(22), 2937-2944 (2009)

2. Allenby, N.E.E., Laing, E., Bucca, G., Kierzek, A.M., Smith, C.P.: Diverse control of metabolism and other cellular processes in streptomyces coelicolor by the phop transcription factor: genome-wide identification of in vivo targets. Nucleic Acids Research (2012). DOI 10.1093/nar/gks766

3. Alon, U.: An Introduction to Systems Biology: Design Principles of Biological Circuits. Chapman \& Hall/CRC (2006)

4. Alon, U.: Network motifs: theory and experimental approaches. Nat. Rev. Genet. 8, 450 - 461 (2007). DOI http://dx.doi.org/10.1038/nrg2102

5. Alvarez-benitez, J.E., Everson, R.M., Fieldsend, J.E.: A mopso algorithm based exclusively on pareto dominance concepts. In: In Third International Conference on Evolutionary MultiCriterion Optimization, EMO 2005, pp. 459-473. SpringerVerlag (2005)

6. Ando, S., Iba, H.: Inference of gene regulatory model by genetic algorithms. In: Evolutionary Computation, 2001. Proceedings of the 2001 Congress on, vol. 1, pp. $712-719$ vol. 1 (2001). DOI 10.1109/CEC.2001.934461

7. Bansal, M., Belcastro, V., Ambesi-Impiombato, A., di Bernardo, D.: How to infer gene networks from expression profiles. Mol Syst Biol 3 (2007)

8. Bansal, M., Gatta, G.D., di Bernardo, D.: Inference of gene regulatory networks and compound mode of action from time course gene expression profiles. Bioinformatics 22(7), 815-822 (2006)

9. Beal, M.J., Falciani, F., Ghahramani, Z., Rangel, C., Wild, D.L.: A bayesian approach to reconstructing genetic regulatory networks with hidden factors. Bioinformatics 21(3), 349-356 (2005)

10. Bell, G.: The Fourth Paradigm: Data-Intensive Scientific Discovery, 1st edn., chap. Foreword, pp. xixv. Microscoft Research (2009)

11. di Bernardo, D., Thompson, M.J., Gardner, T.S., Chobot, S.E., Eastwood, E.L., Wojtovich, A.P., Elliott, S.J., Schaus, S.E., Collins, J.J.: Chemogenomic profiling on a genome-wide scale using reverseengineered gene networks. Nature Biotechnology (3), 377383 (2005)

12. Beyer, H.G., Schwefel, H.P.: Evolution strategies a comprehensive introduction. Natural Computing 1(1), 3-52 (2002). DOI 10.1023/A:1015059928466. URL http://dx.doi.org/10.1023/A:1015059928466

13. for Biotechnology Information, G.N.C.: Genetic sequence data bank (June 15 2013). URL ftp://ftp.ncbi.nih.gov/genbank/gbrel.txt

14. Brockhoff, D., Friedrich, T., Hebbinghaus, N., Klein, C., Neumann, F., Zitzler, E.: Do additional objectives make a problem harder? In: Proceedings of the 9th annual conference on Genetic and evolutionary computation, GECCO '07, pp. 765-772. ACM, New York, NY, USA
(2007). DOI 10.1145/1276958.1277114. URL http://doi.acm.org/10.1145/1276958.1277114

15. Brockhoff, D., Friedrich, T., Hebbinghaus, N., Klein, C., Neumann, F., Zitzler, E.: On the effects of adding objectives to plateau functions. Evolutionary Computation, IEEE Transactions on 13(3), 591 -603 (2009). DOI 10.1109/TEVC.2008.2009064

16. Cai, X.: A multi-objective gp-pso hybrid algorithm for gene regulatroy network modeling. Ph.D. thesis, Kansas State University, Manhatten, Kansas (2009)

17. Cai, X., Koduru, P., Das, S., Welch, S.M.: Simultaneous structure discovery and parameter estimation in gene networks using a multi\&\#45;objective gp\&\#45;pso hybrid approach. Int. J. Bioinformatics Res. Appl. 5(3), 254-268 (2009). DOI 10.1504/IJBRA.2009.026418. URL http://dx.doi.org/10.1504/IJBRA.2009.026418

18. Chen, B.S., Hsu, C.Y., Liou, J.J.: Robust design of biological circuits: Evolutionary systems biology approach. Journal of Biomedicine and Biotechnology (2011)

19. Chen, L.: Computational systems biology on networksand dynamics. In: Optimization and Systems Biology, pp. 5-12 (2007)

20. Chiquet, J., Grandvalet, Y., Ambroise, C.: Inferring multiple graphical structures. Statistics and Computing 21(4), 537-553 (2011)

21. Chowdhury, A., Chetty, M., Vinh, X.: On the reconstruction of genetic network from partial microarray data. In: T. Huang, Z. Zeng, C. Li, C. Leung (eds.) Neural Information Processing, Lecture Notes in Computer Science, vol. 7663, pp. 689-696. Springer Berlin Heidelberg (2012)

22. Crombach, A., Hogeweg, P.: Evolution of evolvability in gene regulatory networks. PLoS Comput Biol 4(7), e1000,112 (2008). DOI 10.1371/journal.pcbi.1000112. URL http://dx.plos.org/10.1371\%2Fjournal.pcbi.1000112

23. von Dassow, G., Meir, E., Munro, E.M., Odell, G.M.: The segment polarity network is a robust developmental module. Nature (2000)

24. De La Fuente, A., Bing, N., Hoeschele, I., Mendes, P.: Discovery of meaningful associations in genomic data using partial correlation coefficients. Bioinformatics 20(18), 3565-3574 (2004)

25. Deb, K.: Multi-Objective Optimisation using Evolutionary Algortihms, 1st edn. Wiley, Kanpur, India (2001)

26. Deb, K., Pratap, A., Agarwal, S., Meyarivan, T.: A fast and elitist multiobjective genetic algorithm: Nsga-ii. Evolutionary Computation, IEEE Transactions on $\mathbf{6}(2), 182-197$ (2002). DOI $10.1109 / 4235.996017$

27. Deb, K., Srinivasan, A.: Innovization: innovating design principles through optimization. In: Proceedings of the 8th annual conference on Genetic and evolutionary computation, GECCO '06, pp. 1629 1636. ACM, New York, NY, USA (2006)

28. Dorigo, M., Birattari, M., Stutzle, T.: Ant colony optimization. Computational Intelligence Magazine, 
IEEE 1(4), 28-39 (2006)

29. DSouza, R.G.L., Sekaran, K.C., , Kandasamy, A.: A phenomic algorithm for reconstruction of gene networks. World Academy of Science, Engineering and Technology 31, 53-58 (2007)

30. D'Souza, R.G.L., Sekaran, K.C., A, K.: Reconstruction of gene networks using phenomic algorithms. International Journal of Artificial Intelligence \& Applications 1(2) (2010)

31. èbre, S.: Inferring dynamic genetic networks with low order independencies. Statistical Applications in Genetics and Molecular Biology 8, 1-38 (2009)

32. Fioravanti, F., Helmer-Citterich, M., Nardelli, E.: Modeling gene regulatory network motifs using statecharts. BMC Bioinformatics 13(4), 1-12 (2012)

33. Gardner, T.S., di Bernardo, D., Lorenz, D., Collins, J.J.: Inferring genetic networks and identifying compound mode of action via expression profiling. Science 301(5629), 102-105 (2003)

34. Geier, F., Timmer, J., Fleck, C.: Reconstructing gene-regulatory networks from time series, knockout data, and prior knowledge. BMC Systems Biology 1 (2007)

35. Glover, F.: Informs Journal on Computing - INFORMS 1(3), 190-206 (1989)

36. Gonze, D.: Coupling oscillations and switches in genetic networks. Biosystems 99(1), 60 - 69 (2010)

37. Hache, H., Lehrach, H., Herwig, R.: Reverse engineering of gene regulatory networks: a comparative study. EURASIP J. Bioinformatics Syst. Biol. 2009, 8:1-8:12 (2009). DOI 10.1155/2009/617281. URL http://dx.doi.org/10.1155/2009/617281

38. Hache, H., Wierling, C., Lehrach, H., Herwig, R.: Reconstruction and validation of gene regulatory networks with neural networks. pp. 319-324 (2007)

39. Hadka, D., Reed, P.: Borg: An auto-adaptive manyobjective evolutionary computing framework. Evolutionary Computation 21, 231-259 (2013)

40. Hallinan, J.: Gene Networks and Evolutionary Computation, pp. 67-96. John Wiley Sons, Inc. (2007). DOI 10.1002/9780470199091.ch4. URL http://dx.doi.org/10.1002/9780470199091.ch4

41. Handl, J., Kell, D.B., Knowles, J.: Multiobjective optimization in bioinformatics and computational biology. IEEE/ACM Trans. Comput. Biol. Bioinformatics 4(2), 279-292 (2007). DOI 10.1109/TCBB.2007.070203. URL http://dx.doi.org/10.1109/TCBB.2007.070203

42. Handl, J., Lovell, S.C., Knowles, J.: Investigations into the effect of multiobjectivization in protein structure prediction. In: Proceedings of the 10th international conference on Parallel Problem Solving from Nature: PPSN X, pp. 702-711. SpringerVerlag, Berlin, Heidelberg (2008)

43. Hartwell, L.H., Hopfield, J.J., Leibler, S., Murray, A.W.: From molecular to modular cell biology. Nature 402, C47-C52 (1999)

44. Hecker, M., Lambeck, S., Toepfer, S., van Someren, E., Guthke, R.: Gene regulatory network inference: Data integration in dynamic modelsa review.
Biosystems 96(1), 86 - 103 (2009)

45. Hey, T., Tansley, S., Tolle, K. (eds.): The Fourth Paradigm: Data-Intensive Scientific Discovery, 1st edn. Microscoft Research (2009)

46. Higuera, C., Villaverde, A.F., Banga, J.R., Ross, J., Morn, F.: Multi-criteria optimization of regulation in metabolic networks. PLoS ONE 7(7), e41,122 (2012)

47. Hoon, M.D., Imoto, S., Miyano, S.: Inferring gene regulatory networks from time-ordered gene expression data of bacillus subtilis using differential equations. In: Pac. Symp. Biocomput, pp. 17-28 (2003)

48. Iba, H., Mimura, A.: Inference of a gene regulatory network by means of interactive evolutionary computing. Information Sciences 145(34), 225 236 (2002). DOI http://dx.doi.org/10.1016/S00200255(02)00234-7

49. IBM: What is big data? $(3 / 7 / 13)$. URL http://www-01.ibm.com/software/data/bigdata/

50. Ishibuchi, H., Tsukamoto, N., Nojima, Y.: Evolutionary many-objective optimization: A short review. In: Evolutionary Computation, 2008. CEC 2008. (IEEE World Congress on Computational Intelligence). IEEE Congress on, pp. 2419-2426 (2008)

51. Ishibuchi, H., Tsukamoto, N., Nojima, Y.: Evolutionary many-objective optimization: A short review. In: Proceedings of Congress on Evolutionary Computation, CEC, pp. 2424-2431 (2008)

52. Jain, H., Deb, K.: An improved adaptive approach for elitist nondominated sorting genetic algorithm for many-objective optimization. In: R. Purshouse, P. Fleming, C. Fonseca, S. Greco, J. Shaw (eds.) Evolutionary Multi-Criterion Optimization, Lecture Notes in Computer Science, vol. 7811, pp. 307321. Springer Berlin Heidelberg (2013). DOI 10.1007/978-3-642-37140-0_25

53. Jensen, M.T.: Helper-objectives: Using multiobjective evolutionary algorithms for singleobjective optimisation. Journal of Mathematical Modelling and Algorithms 3, 323-347 (2004). 10.1023/B:JMMA.0000049378.57591.c6

54. Jin, Y., Meng, Y.: Emergence of robust regulatory motifs from in silico evolution of sustained oscillation. Biosystems 103(1), 38 - 44 (2011)

55. de Jong, H., Geiselmann, J.: Modeling and simulation of genetic regulatory networks by ordinary differential equations. In: Genomic Signal Processing and Statistics, p. 201239. Hindawi Publishing Corporation, New York (2005)

56. Kabir, M., Noman, N., Iba, H.: Reverse engineering gene regulatory network from microarray data using linear time-variant model. BMC Bioinformatics 11, S56 (2010)

57. KARABOGA, D.: An idea based on honey bee swarm for numerical optimization. Tech. rep., Erciyes University, Engineering Faculty, Computer Engineering Department, Kayseri/Türkiye

58. Karlebach, G., Shamir, R.: Modelling and analysis of gene regulatory networks. Nat Rev Mol Cell Biol 9, 770 - 780. DOI http://dx.doi.org/10.1038/nrm2503 
59. Keedwell, E., Narayanan, A.: Discovering gene networks with a neural-genetic hybrid. IEEE/ACM Trans. Comput. Biol. Bioinformatics 2(3), 231242 (2005). DOI 10.1109/TCBB.2005.40. URL http://dx.doi.org/10.1109/TCBB.2005.40

60. Kennedy, J., Eberhart, R.: Particle swarm optimization. In: Neural Networks, 1995. Proceedings., IEEE International Conference on, vol. 4, pp. 1942-1948 vol.4 (1995)

61. Khammash, M.: Reverse engineering: the architecture of biological networks. BioTechniques 44, 323,329 (2008)

62. Khammash, M., El-Samad, H.: Systems biology: from physiology to gene regulation. Control Systems, IEEE 24(4), 62-76 (2004). DOI 10.1109/MCS.2004.1316654

63. Kikuchi, S., Tominaga, D., Arita, M., Takahashi, K., Tomita, M.: Dynamic modeling of genetic networks using genetic algorithm and s-system. Bioinformatics 19(5), 643-650 (2003). DOI 10.1093/bioinformatics/btg027

64. Kirkpatrick, S., Gelatt, C.D., Vecchi, M.P.: Optimization by simulated annealing. Science 220, 671680 (1983)

65. Klemm, S.: Causal structure identication in nonlinear dynamical systems (2008)

66. Knowles, J.D., Corne, D.W.: Approximating the nondominated front using the pareto archived evolution strategy. Evol. Comput. 8(2), 149-172 (2000)

67. Knowles, J.D., Watson, R.A., Corne, D.: Reducing local optima in single-objective problems by multi-objectivization. In: Proceedings of the First International Conference on Evolutionary MultiCriterion Optimization, EMO '01, pp. 269-283. Springer-Verlag, London, UK, UK (2001). URL http://dl.acm.org/citation.cfm?id=647889.736521

68. Kukkonen, S., Lampinen, J.: Gde3: the third evolution step of generalized differential evolution. In: Evolutionary Computation, 2005. The 2005 IEEE Congress on, vol. 1, pp. 443-450 Vol.1 (2005)

69. Lanely, D.: 3d data management: Controlling data volume, velocity and variety. Tech. rep., Application Delivery strategies: META Group (2001)

70. Larus, J., Gannon, D.: The Fourth Paradigm: DataIntensive Scientific Discovery, 1st edn., chap. Multicore Computing and Scientific Discovery, pp. 125129. Microscoft Research (2009)

71. Laumanns, M., Rudolph, G., Schwefel, H.P.: A spatial predator-prey approach to multi-objective optimization: A preliminary study. In: Proceedings of the 5th International Conference on Parallel Problem Solving from Nature, PPSN V, pp. 241-249. Springer-Verlag, London, UK, UK (1998)

72. Leclerc, R.D.: Survival of the sparsest: robust gene networks are parsimonious. Molecular Systems Biology pp. 1-6 (2008)

73. Lee, W.P., Hsiao, Y.T.: Inferring gene regulatory networks by incremental evolution and network decomposition. In: Optimization and Systems Biology, pp. 311-324 (2008)
74. Lenser, T., Hinze, T., Ibrahim, B., Dittrich, P.: Towards evolutionary network reconstruction tools for systems biology. In: E. Marchiori, J. Moore, J. Rajapakse (eds.) Evolutionary Computation,Machine Learning and Data Mining in Bioinformatics, Lecture Notes in Computer Science, vol. 4447, pp. 132-142. Springer Berlin Heidelberg (2007). DOI 10.1007/978-3-540-71783-6_13. URL http://dx.doi.org/10.1007/978-3-540-71783-6_13

75. Li, C., Chen, L., Aihara, K.: A systems biology perspective on signal processing in genetic network motifs [life sciences]. Signal Processing Magazine, IEEE 24(2), $136-147$ (2007). DOI 10.1109/MSP.2007.323279

76. Li, J., Zhang, X.S.: An optimization model for gene regulatory network reconstruction with known biological information. In: Optimization and Systems Biology, pp. 35-44 (2007)

77. Liu, Y., Niculescu-Mizil, A., Lozano, A.C., Lu, Y.: Temporal graphical models for cross-species gene regulatory network discovery. J. Bioinformatics and Computational Biology 9(2), 231-250 (2011)

78. Lochtefeld, D., Ciarallo, F.: Multiobjectivization via helper-objectives with the tunable objectives problem. Evolutionary Computation, IEEE Transactions on $\mathbf{1 6}(3), \quad 373-390$ (2012). DOI 10.1109/TEVC.2011.2136345

79. Maki, Y., Tominaga, D., Okamoto, M., Watanabe, S., Eguchi, Y.: Development of a system for the inference of large scale genetic networks. Pac Symp Biocomput. pp. 446-458 (2001)

80. Margolin, A.A., Nemenman, I., Basso, K., Wiggins, C., Stolovitzky, G., Favera, R.D., Califano, A.: ARACNE: An algorithm for the reconstruction of gene regulatory networks in a mammalian cellular context. BMC Bioinformatics 7((Suppl 1)), S7 (2006)

81. McLachlan, G.J., Do, K.A., Ambroise, C.: Analyzing Microarray gene Expression Data. WileyInterscience (2004)

82. Mendoza, M.R., Bazzan, A.L.C.: Evolving random boolean networks with genetic algorithms for regulatory networks reconstruction. In: Proceedings of the 13th annual conference on Genetic and evolutionary computation, GECCO '11, pp. 291-298. ACM, New York, NY, USA (2011). DOI 10.1145/2001576.2001617. URL http://doi.acm.org/10.1145/2001576.2001617

83. Milo, R., Shen-Orr, S., Itzkovitz, S., Kashtan, N., Chklovskii, D., Alon, U.: Network motifs: Simple building blocks of complex networks. Science 298(5594), 824-827 (2002). DOI 10.1126 /science.298.5594.824

84. Mitchell, M.: An Introduction to Genetic Algorithms. The MIT Press (1999)

85. Mondal, B., Sarkar, A., Hasan, M., Noman, N.: Reconstruction of gene regulatory networks using differential evolution. In: Computer and Information Technology (ICCIT), 2010 13th International Conference on, pp. $440-445$ (2010). DOI 


\subsection{9/ICCITECHN.2010.5723898}

86. Morishita, R., Imade, H., Ono, l., Ono, N., Okamoto, M.: Finding multiple solutions based on an evolutionary algorithm for inference of genetic networks by s-system. In: Evolutionary Computation, 2003. CEC '03. The 2003 Congress on, vol. 1, pp. 615-622 Vol.1 (2003)

87. Nakayama, T., Seno, S., Matsuda, H.: Inference of s-system models of gene regulatory networks using immune algorithm. J Bioinform Comput Biol 9, 75$86(2011)$

88. Noman, N., Iba, H.: Inference of genetic networks using s-system: information criteria for model selection. In: Proceedings of the 8th annual conference on Genetic and evolutionary computation, GECCO '06, pp. 263-270. ACM, New York, NY, USA (2006)

89. Noman, N., Iba, H.: Inferring gene regulatory networks using differential evolution with local search heuristics. IEEE/ACM Trans. Comput. Biol. Bioinformatics 4(4), 634-647 (2007)

90. Noor, A., Serpedin, E., Nounou, M., Nounou, H., Mohamed, N., Chouchane, L.: Information theoretic methods for modeling of gene regulatory networks. In: Computational Intelligence in Bioinformatics and Computational Biology (CIBCB), 2012 IEEE Symposium on, pp. 418-423 (2012). DOI 10.1109/CIBCB.2012.6217260

91. Penfold, C.A., Wild, D.L.: How to infer gene networks from expression profiles, revisited. Interface Focus (2011)

92. Purshouse, R.C., Fleming, P.J., Fonseca, C.M., Greco, S., Shaw, J.: 7th international conference, emo 2013, sheffield, uk, march 19-22, 2013. proceedings. In: Evolutionary Multi-Criterion Optimization, vol. 7811. Springer Berlin Heidelberg (2013)

93. Quackenbush, J.: Computational analysis of microarray data. Nature Reviews Genetics (6), 418427 (2001)

94. Ramons, A.F., Innocentini, G., Forger, F.M., Hornos, J.E.: Symmetry in biology: from genetic code to stochastic gene regulation. IET Syst Biol 4(5), 311-329 (2010). DOI 10.1049/ietsyb. 2010.0058

95. Rangel, C., Angus, J., Ghahramani, Z., Lioumi, M., Sotheran, E., Gaiba, A., Wild, D.L., Falciani, F.: Modeling t-cell activation using gene expression profiling and state-space models. Bioinformatics 20(9), 1361-1372 (2004)

96. Rau, A., Jaffrzic, F., Foulley, J.L., Doerge, R.: Reverse engineering gene regulatory networks using approximate bayesian computation. Statistics and Computing 22(6), 1257-1271 (2012)

97. Sakamoto, E., Iba, H.: Evolutionary Inference of a Biological Network as Differential Equations by Genetic Programming. Genome Informatics pp. 276 277 (2001)

98. Samad, H.E., Khammash, M., Petzold, L., Gillespie, D.: Stochastic modelling of gene regulatory networks. Int. J. Robust Nonlinear Control
99. Savageau, M.A.: Journal of Theoretical Biology 25, 370-379 (1969)

100. Schäfer, J., Strimmer, K.: An empirical bayes approach to inferring large-scale gene association networks. Bioinformatics 21(6), 754-764 (2005)

101. Schmidt, M., Lipson, H.: Distilling free-form natural laws from experimental data. Science 324(5923), 81-85 (2009)

102. Schwefel, H.P.: Numerical optimization of computer models. Chichester: Wiley Sons (1981)

103. Seth, A.K.: A $\{$ MATLAB $\}$ toolbox for granger causal connectivity analysis. Journal of Neuroscience Methods 186(2), 262 - 273 (2010)

104. Shen-Orr, S.S., Milo, R., Mangan, S., Alon, U.: Network motifs in the transcriptional regulations network of escherichia coli. Nature Genetics 31, 64-68 (2002). DOI 10.1028/ng881

105. Shin, A., Iba, H.: Construction of genetic network using evolutionary algorithm and combined fitness function. Genome Inform 14, 2003 (2003)

106. Sîrbu, A., Ruskin, H., Crane, M.: Integrating heterogeneous gene expression data for gene regulatory network modelling. Theory in Biosciences 131(2), 95-102 (2012)

107. Sirbu, A., Ruskin, H.J., Crane, M.: Comparison of evolutionary algorithms in gene regulatory network model inference. BMC Bioinformatics 11, 59 (2010)

108. Sîrbu, A., Ruskin, H.J., Crane, M.: Cross-platform microarray data normalisation for regulatory network inference. PLoS ONE 5(11), e13,822 (2010)

109. Sîrbu, A., Ruskin, H.J., Crane, M.: Stages of gene regulatory network inference: the evolutionary algorithm role. In: P.E. Kita (ed.) Evolutionary Algorithms. InTech (2011)

110. Solé, R.V., Valverde, S.: Are network motifs the spandrels of cellular complexity? Trends in Ecology and amp; Evolution (8), 419 - 422. DOI 10.1016/j.tree.2006.05.013

111. de Sompel, H.V., Lagoze, C.: The Fourth Paradigm: Data-Intensive Scientific Discovery, 1st edn., chap. All Aboard: Toward a Machine-Friendly Scholarly Communication System, pp. 193-199. Microscoft Research (2009)

112. Spieth, C., Streichert, F., Speer, N., Zell, A.: Optimizing topology and parameters of gene regulatory network models from time-series experiments. In: K. Deb (ed.) Genetic and Evolutionary Computation GECCO 2004, Lecture Notes in Computer Science, vol. 3102, pp. 461-470. Springer Berlin Heidelberg (2004)

113. Spieth, C., Streichert, F., Supper, J., Speer, N., Zell, A.: Feedback memetic algorithms for modeling gene regulatory networks. In: Computational Intelligence in Bioinformatics and Computational Biology, 2005. CIBCB '05. Proceedings of the 2005 IEEE Symposium on, pp. 1-7 (2005)

114. Srinivas, N., Deb, K.: Multiobjective optimization using nondominated sorting in genetic algorithms. Evolutionary Computation 2, 221-248 (1994) 
115. Swain, M., Hunniford, T., Mandel, J., Palfreyman, N., Dubitzky, W.: Modeling gene-regulatory networks using evolutionary algorithms and distributed computing. In: Cluster Computing and the Grid, 2005. CCGrid 2005. IEEE International Symposium on, vol. 1, pp. 512-519 Vol. 1 (2005). DOI 10.1109/CCGRID.2005.1558596

116. Tegnèr, J., Yeung, M.K.S., Hasty, J., Collins, J.J.: Reverse engineering gene networks: Integrating genetic perturbations with dynamical modeling. Proceedings of the National Academy of Sciences 100(10), 5944-5949 (2003)

117. Thomas, S., Jin, Y.: Single and multi-objective in silico evolution of tunable genetic oscillators. In: R. Purshouse, P. Fleming, C. Fonseca, S. Greco, J. Shaw (eds.) Evolutionary Multi-Criterion Optimization, Lecture Notes in Computer Science, vol. 7811, pp. 696-709. Springer Berlin Heidelberg (2013)

118. Thomas, S.A., Jin, Y.: Combining genetic oscillators and switches using evolutionary algorithms. In: Computational Intelligence in Bioinformatics and Computational Biology (CIBCB), 2012 IEEE Symposium on, pp. 28 -34 (2012). DOI 10.1109/CIBCB.2012.6217207

119. Thomas, S.A., Jin, Y.: Evolving connectivity between genetic oscillators and switches using evolutionary algorithms. J. Bioinform. ComputBiol. 11(3:1341001) (2013)

120. Tobin, F.L., Damian-iordache, V., Greller, L.D.: Towards the reconstruction of gene regulatory networks (1999)

121. Tominaga, D., Okamoto, M., Maki, Y., Watanabe, S., Eguchi, Y.: Nonlinear numerical optimization technique based on a genetic algorithm for inverse problems: Towards the inference of genetic networks. In: German Conference on Bioinformatics'99, pp. 127-140 (1999)

122. Vignes, M., Vandel, J., Allouche, D., RamadanAlban, N., Cierco-Ayrolles, C., Schiex, T., Mangin, B., de Givry, S.: Gene regulatory network reconstruction using bayesian networks, the dantzig selector, the lasso and their meta-analysis. PLoS ONE 6(12), e29,165 (2011)

123. Voit, E.O.: Model identifictaion: A key challenge is computational systems biology. In: Optimization and Systems Biology, pp. 1-12 (2008)

124. Voit, E.O., Almeida, J.: Decoupling dynamical systems for pathway identification from metabolic profiles. Bioinformatics 20(11), 1670-1681 (2004)

125. Wang, Y., Zhang, X.S., Chen, L.: Optimization meets systems biology. BMC Systems Biology 4(Suppl 2), 1-4 (2010). DOI 10.1186/1752-0509-4S2-S1. URL http://dx.doi.org/10.1186/1752-05094-S2-S1

126. Whitehead, D.J., Skusa, A., Kennedy, P.J.: Evaluating an Evolutionary Approach for Reconstructing Gene Regulatory Networks. In: Ninth International Conference on the Simulation and Synthesis of Living Systems (ALIFE9). MIT Press, Boston (2004)
127. Wieczorek, M.A., Jolliff, B.L., Khan, A., Pritchard, M.E., Weiss, B.P., Williams, J.G., Hood, L.L., Righter, K., Neal, C.R., Shearer, C.K., McCallum, I.S., Tompkins, S., Hawke, B.R., Peterson, C., Gillis, J.J., Bussey, B.: The constitution and structure of the lunar interior. Reviews in Mineralogy Geochemistry 60, 221-364 (2006)

128. Wiggins, C.: It's an exciting time for data in new york city. University of Columbia Engineering Newsletter (2012)

129. Xiao, M., Zhang, L., He, B., Xie, J., Zhang, W.: A parallel algorithm of constructing gene regulatory networks. In: D.Z. Du, X.S. Zhang (eds.) Optimization and Systems Biology, Lecture Notes in Operations Research, vol. 11, pp. 184-188. WORLD PUBLISHING CORPORATION (2009). URL http://www.aporc.org/LNOR/11/OSB2009C01.pdf

130. Xiong, J., Zhou, T.: Gene regulatory network inference from multifactorial perturbation data using both regression and correlation analyses. PLoS ONE 7(9), e43,819 (2012)

131. Yip, K.Y., Alexander, R.P., Yan, K.K., Gerstein, M.: Improved reconstruction of jitalic ¿in silicoi/italic $\&$ gene regulatory networks by integrating knockout and perturbation data. PLoS ONE 5(1), e8121 (2010). DOI 10.1371/journal.pone.0008121

132. Yu, J., Smith, V.A., Wang, P.P., Hartemink, A.J., Jarvis, E.D.: Advances to bayesian network inference for generating causal networks from observational biological data. Bioinformatics 20(18), 35943603 (2004)

133. Yuan, Y., Stan, G.B., Warnick, S., Goncalves, J.M.: Robust dynamical network structure reconstruction. Automatica, Special Issue on Systems Biology 47, 1230-1235 (2011)

134. Zhang, Q., Li, H.: Moea/d: A multi-objective evolutionary algorithm based on decomposition. IEEE Trans. on Evolutionary Computation 11, 712-731 (2007)

135. Zhang, X., Zhao, X.M., He, K., Lu, L., Cao, Y., Liu, J., Hao, J.K., Liu, Z.P., Chen, L.: Inferring gene regulatory networks from gene expression data by path consistency algorithm based on conditional mutual information. Bioinformatics 28(1), 98-104 (2012)

136. Zhang, Z., Bajic, V.B., Yu, J., Cheung, K.H., Townsend, J.P.: Data integration in bioinformatics: Current efforts and challenges. In: D.M.A. Mahdavi (ed.) Trends and Methodologies, chap. 2, pp. 41-56. InTech

137. Zhu, H., Rao, R.S.P., Zeng, T., Chen, L.: Reconstructing dynamic gene regulatory networks from sample-based transcriptional data. Nucleic Acids Research 40(21), 10,657-10,667 (2012)

138. Zitzler, E., Künzli, S.: Indicator-based selection in multiobjective search. In: in Proc. 8th International Conference on Parallel Problem Solving from Nature (PPSN VIII, pp. 832-842. Springer (2004) 